\title{
Tidal double detonation: a new mechanism for a thermonuclear explosion of a white dwarf induced by a tidal disruption event
}

\author{
Ataru Tanikawa, ${ }^{1,2 \star}$ \\ ${ }^{1}$ Department of Earth Science and Astronomy, College of Arts and Sciences, The University of Tokyo, 3-8-1 Komaba, Meguro-ku, Tokyo 153-8902, \\ ${ }^{2}$ RIKEN Advanced Institute for Computational Science, 7-1-26 Minatojima-minami-machi, Chuo-ku, Kobe, Hyogo 650-0047, Japan
}

Accepted XXX. Received YYY; in original form ZZZ

\begin{abstract}
We suggest "tidal double detonation": a new mechanism for a thermonuclear explosion of a white dwarf (WD) induced by a tidal disruption event (TDE). Tidal detonation is also a WD explosion induced by a TDE. In this case, helium (He) and carbon-oxygen $(\mathrm{CO})$ detonation waves incinerate $\mathrm{He} \mathrm{WD}$ and $\mathrm{CO} \mathrm{WD}$, respectively. On the other hand, for tidal double detonation, He detonation is first excited in the He shell of a CO WD, and drives $\mathrm{CO}$ detonation in the $\mathrm{CO}$ core. We name this mechanism after the double detonation scenario in the context of type Ia supernovae. In this paper, we show tidal double detonation occurs in shallower encounter of a CO WD with an intermediate mass black hole (IMBH) than simple tidal detonation, performing numerical simulations for CO WDs with $0.60 M_{\odot}$ with and without a He shell. We expect tidal double detonation spreads opportunity to WD TDEs illuminating IMBHs.
\end{abstract}

Key words: black hole physics - hydrodynamics - nuclear reactions, nucleosynthesis, abundances - supernovae: general - white dwarfs

\section{INTRODUCTION}

A tidal disruption event (TDE) is a phenomenon in which a star is tidally torn apart by a black hole (BH). There are many TDE candidates in which main sequence (MS) stars are disrupted by massive black holes (MBHs) (e.g. Komossa 2015; Auchettl et al. 2017). White dwarfs (WDs) are also expected to experience TDEs. It is not by MBHs but by intermediate mass black holes (IMBHs) that WDs are disrupted (Luminet \& Pichon 1989). WD TDEs will produce not only bright flares powered by accretion of WD debris onto IMBHs, but also thermonuclear emissions of radioactive nuclei synthesized by tidal detonation (Luminet \& Pichon 1989; Wilson \& Mathews 2004; Rosswog et al. 2008, 2009). WD TDEs can be probes to explore IMBHs.

Tidal detonation happens in a WD TDE as follows. A WD closely passes by an IMBH, and is stretched by the tidal field of the IMBH in the direction of the orbital plane (hereafter $x$ - $y$ plane). On the other hand, the WD is compressed in the direction perpendicular to the $x-y$ plane (hereafter $z$-direction). This is because the WD size become comparable to the separation between the WD and IMBH at the pericenter. The compression of the WD has to accom-

^ E-mail: tanikawa@ea.c.u-tokyo.ac.jp pany shock heating for tidal detonation. In other words, adiabatic compression is insufficient for tidal detonation. Although Luminet \& Pichon (1989) have suggested a helium (He) WD can be detonated only by adiabatic compression, their He WD model has relatively larger mass $\left(0.6 M_{\odot}\right)$ or higher density $\left(\sim 10^{7} \mathrm{~g} \mathrm{~cm}^{-3}\right)$ than in reality (e.g. Parsons et al. 2017). Wilson \& Mathews (2004) have reported a carbon-oxygen $(\mathrm{CO}) \mathrm{WD}$ can experience tidal detonation by adiabatic compression. However, they have not taken into account elongation of the CO WD by a tidal field of a $\mathrm{BH}$, and have overestimated the density of the CO WD. Note that nuclear reactions are more active under higher density environment.

In Tanikawa et al. (2017) (Paper I) and Tanikawa (2017) (Paper II), we have investigated tidal detonation triggered by a shock wave. We have found the following three facts. First, the shock wave does not always result in a detonation wave. Second, the shock wave is easier to excite a detonation wave under higher density environment and in lighter nuclear compositions (in the order of $\mathrm{He}, \mathrm{CO}$, and oxygen-neon-magnesium (ONeMg) compositions). Third, the shock wave arises near the surface of a $\mathrm{WD}$, when the ratio of the tidal disruption radius to the pericenter distance $(\beta)$ is not so large. These facts imply a shock wave triggers tidal detonation more easily from a 
CO WD (ONeMg WD) with a He shell than from a CO WD (ONeMg WD) without a He shell. This tidal detonation proceeds as follows. A shock wave generates He detonation in the He shell of a WD. The He detonation invades into the $\mathrm{CO}$ core of the $\mathrm{WD}$, and ignites $\mathrm{CO}$ detonation in the $\mathrm{CO}$ core. We name this explosion mechanism "tidal double detonation" after the double detonation scenario of type Ia supernovae (Nomoto 1980, 1982; Woosley et al. 1980).

The tidal double detonation is fairly a new scenario for a thermonuclear explosion of a WD by a TDE. Tidal detonation considers only He detonation in He WD and only $\mathrm{CO}$ detonation in CO WD (e.g. Rosswog et al. $2008,2009)$. There are various double detonation scenarios for a thermonuclear explosion of a WD (Nomoto 1980, 1982; Woosley et al. 1980; Livne 1990; Bildsten et al. 2007; Guillochon et al. 2010; Shen et al. 2010; Pakmor et al. 2013). However, in these scenarios, He detonation is triggered by accretion of matter from a companion star onto a WD.

In this paper, we show the tidal double detonation works better for a thermonuclear explosion of a CO WD than simple tidal detonation, if a WD has a He shell whose mass fraction is $5 \%$ of the WD. Note that a CO WD can have a He shell whose mass fraction is several \% (Iben \& Tutukov 1985, 1993; Salaris et al. 2000).

The structure of this paper is as follows. We describe our method in section 2. We present our results in section 3 . Finally, we make a conclusion in section 4 .

\section{METHOD}

We investigate tidal detonation and tidal double detonation in a similar way to Paper II. We follow overall evolution of a WD by 3-dimensional (3D) smoothed particle hydrodynamics (SPH) simulation. We extract profiles of density and $z$-velocity in the $z$-direction from a portion of the WD, and use the profiles for an initial condition of 1-dimensional (1D) mesh simulation. We perform 1D mesh simulation, and follow tidal detonation and tidal double detonation. We combine 1D mesh simulation with 3D SPH simulation in order to avoid spurious heating due to low space resolution (Rosswog et al. 2008, 2009, Paper I), and in order to resolve a shock wave near the surface of a WD.

Our 3D SPH code is the same as in Paper II. We use FDPS (Iwasawa et al. 2016) for parallelization of our 3D SPH code. We adopt the Helmholtz equation of state (EoS) (Timmes \& Swesty 2000) which is calculated in the routine developed by the Center for Astrophysical Thermonuclear Flashes at the University of Chicago. We do not take into account nuclear reactions in 3D SPH simulation. We follow the evolution of a CO WD with $0.6 M_{\odot}$ by $3 \mathrm{D} \mathrm{SPH}$ simulation. The composition is $50 \%$ carbon and $50 \%$ oxygen in mass. The CO WD has no He shell. The number of SPH particles, $N$, is 100 millions. We relax the configure of $\mathrm{SPH}$ particles in the same way as in Tanikawa et al. (2015). The IMBH mass is $300 M_{\odot}$. We adopt Newtonian potential for the IMBH gravity. The orbit of the WD around the IMBH is parabolic. The ratio of the tidal disruption distance to the pericenter distance $(\beta)$ is 5 . The IMBH does not irrupt into nor swallow the WD even if we consider general relativistic effects for the IMBH gravity (Tejeda \& Rosswog 2013). The

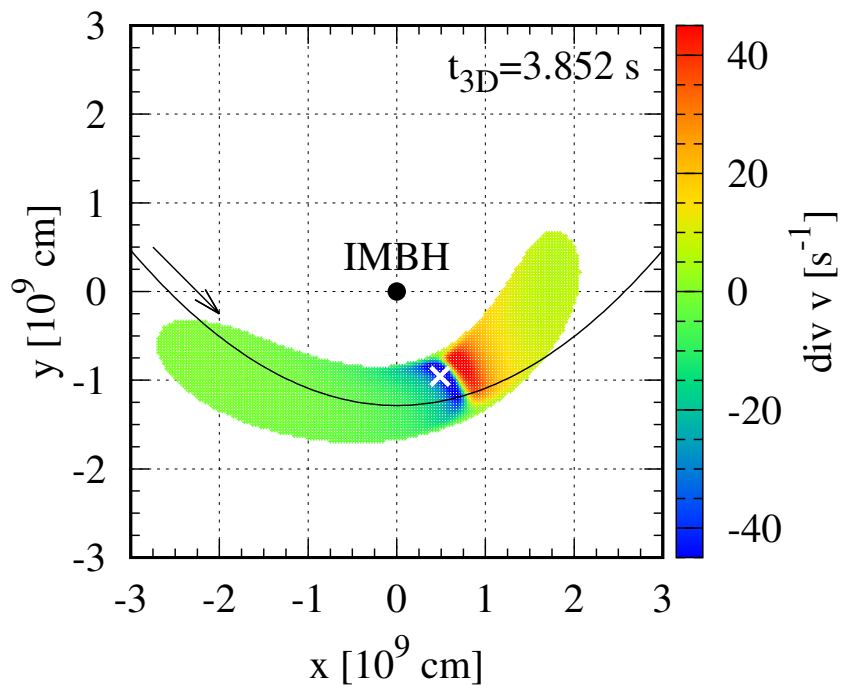

Figure 1. Divergence of velocity on the $x-y$ plane at the indicated time in the CO WD. The IMBH is located at the coordinate origin. The solid curve shows the orbit of the WD on the assumption that the WD is a point mass, and the arrow indicates the traveling direction of the WD orbit. The white cross indicates the extracted portion.

WD passes the pericenter from $t_{3 \mathrm{D}}=3.5 \mathrm{~s}$ to $t_{3 \mathrm{D}}=4.5 \mathrm{~s}$, where $t_{3 \mathrm{D}}$ is the time from the starting time of the $3 \mathrm{D} \mathrm{SPH}$ simulation.

We make 1D initial conditions, extracting density and zvelocity profiles in the $z$-direction from a portion of the WD simulated by the above 3D SPH method. We choose the portion indicated by the white cross in Figure 1. The portion will bounce back immediately in the subsequent evolution. Owing to our choice of the portion, we minimize the difference between $1 \mathrm{D}$ and $3 \mathrm{D}$ simulation results which comes from 3D effects, such as a tidal field of the IMBH. This is because we can follow the evolution of the portion for as short a term as possible. Here, the evolution of the portion is such that a pressure wave is generated at the bounce time, the pressure wave steepens into a shock wave, and finally the shock wave excites a detonation wave.

Using 3D SPH results of the CO WD, we obtain density and $z$-velocity profiles in the $z$-direction in the portion by applying SPH kernel interpolation. We set profiles of nuclear elements as follows. For a CO WD without a He shell, we assign only the $\mathrm{CO}$ composition to all the regions for a $1 \mathrm{D}$ initial condition. For a CO WD with a He shell, we assign the $\mathrm{CO}$ composition to the inner region and the He composition $(100 \% \mathrm{He}$ in mass) to the outer region. The mass fraction of the outer region is $1 \%(2 \%)$. This is because we find the outermost $1 \%$ (2\%) SPH particles in this portion form a He shell in the 3D SPH simulation, assuming that the outermost $5 \%(10 \%) \mathrm{SPH}$ particles in the initial CO WD form a He shell in the 3D SPH simulation. The mass fraction of $\mathrm{He}$ composition is much less than 5\% (10\%), since the geometry of the extracted portion is planar, not spherical.

For 1D mesh simulation, we use the FLASH code (Fryxell et al. 2000). We use uniform mesh. We adopt the Helmholtz EoS and Aprox13 (Timmes et al. 2000) for our EoS and nuclear reaction networks, respectively. We suppress nuclear burning in shocked cells. The calculation do- 
main geometry is planar. The domain range is $0 \leq z / 10^{8} \mathrm{~cm} \leq$ 1.0. The number of mesh is 6400 . The boundary condition is the reflection condition at $z=0 \mathrm{~cm}$ and the outflow condition at $z / 10^{8} \mathrm{~cm}=1.0$. The time from the starting time of $1 \mathrm{D}$ mesh simulation is indicated by $t_{1 \mathrm{D}}$.

\section{RESULTS}

We verify the evolution in the $1 \mathrm{D}$ mesh simulation mirrors the evolution of the column in the 3D SPH simulation until a shock wave appears. Figure 2 shows the time evolution of density, pressure, and z-velocity in 1D mesh simulation for a CO WD without a He shell, and in $3 \mathrm{D} \mathrm{SPH}$ simulation. At $t_{1 \mathrm{D}}=0 \mathrm{~s}, z$-velocity gradient is positive at $z \gtrsim 3 \times 10^{7} \mathrm{~cm}$, since we set the $z$-velocity to be zero outside the WD. At $t_{1 \mathrm{D}} \sim 0.0234 \mathrm{~s}$, the portion bounces back, and at $t_{1 \mathrm{D}} \sim 0.0376 \mathrm{~s}$, a shock wave appears. The evolution of physical quantities in the 1D mesh simulation is in good agreement with the evolution of physical quantities in the 3D SPH simulation. Despite that the 1D mesh simulation ignores 3D effects, such as a tidal field, density and pressure in the 1D mesh simulation are larger than those in the 3D SPH simulation by $10 \%$ at $z=0$, and by $20 \%$ at the edge of the WD when the shock wave appears at $t_{1 \mathrm{D}} \sim 0.0376 \mathrm{~s}$. The $z$-velocity in the $1 \mathrm{D}$ mesh simulation evolves in the same way as that in the 3D SPH simulation. Later, we discuss whether the density overestimate affects the initiation of a He detonation wave, or not.

Figure 3 shows the time evolution in 1D mesh simulation for a CO WD with a He shell. The mass fraction of the He shell is $2 \%$. In order to show tidal double detonation clearly, we present the case where the mass fraction of the $\mathrm{He}$ shell is $2 \%$. From the beginning, the WD shrinks to the $x-y$ plane (or $z=0$ ). At $t_{1 \mathrm{D}} \sim 0.0234 \mathrm{~s}$, it bounces back, and a pressure wave arises. At $t_{1 \mathrm{D}} \sim 0.0376 \mathrm{~s}$, the pressure wave steepens into a shock wave (see the middle panel at $t_{1 \mathrm{D}}=0.0376 \mathrm{~s}$ ), and the shock wave triggers explosive nuclear reactions in the He shell (see the bottom panel at $t_{1 \mathrm{D}}=0.0376 \mathrm{~s}$ ). The explosive nuclear reactions generate a reverse shock wave (see the middle panel at $t_{1 \mathrm{D}}=0.0381 \mathrm{~s}$ ). The reverse shock wave accompanies He detonation which consumes large amounts of He materials (see the bottom panel at $\left.t_{1 \mathrm{D}}=0.0381 \mathrm{~s}\right)$. The He detonation invades into the $\mathrm{CO}$ core, and achieves $\mathrm{CO}$ detonation in the $\mathrm{CO}$ core. In fact, the $\mathrm{CO}$ detonation burns out large amounts of $\mathrm{C}$ materials (see the bottom panel at $t_{1 \mathrm{D}}=0.0430 \mathrm{~s}$ ). We also obtain the same results in the case where the mass fraction of a He shell is $1 \%$. Therefore, the CO WD with a He shell whose mass fraction is at most $5 \%$ succeeds tidal double detonation.

We investigate the initiation point of the He detonation in detail. In Figure 4, we zoom in on the position where the He detonation starts. We can see the mass fraction of ${ }^{4} \mathrm{He}$ at $z \sim 1.166 \times 10^{7} \mathrm{~cm}$ is smaller than at its surroundings. This is the initiation point of the He detonation. The shock wave generating the He detonation is between the two vertical dotted lines. Explosive nuclear reactions occur behind the shock wave. This is because we suppress nuclear reaction networks in shocked cells. The mass fraction of ${ }^{4} \mathrm{He}$ is unity at the surroundings of the initiation point of the $\mathrm{He}$ detonation. Therefore, the He detonation starts under pure
He environment. Even if the components of the He shell and $\mathrm{CO}$ core are mixed, the mixing does not affect the initiation of the He detonation.

Figure 5 shows the time evolution in $1 \mathrm{D}$ mesh simulation for a CO WD without a He shell. The evolution of the WD is the same as a CO WD with a He shell from the beginning until the emergence of a shock wave $\left(t_{1 \mathrm{D}}=0.0376 \mathrm{~s}\right)$. However, the shock wave excites nuclear reactions slightly, and consumes small amounts of $\mathrm{C}$ materials (see the bottom panel at $t_{1 \mathrm{D}}=0.0430 \mathrm{~s}$ ). The nuclear reactions cannot trigger further nuclear reactions. The nuclear reactions cease soon. This CO WD fails simple tidal detonation.

The shock wave successfully excites He detonation in the $\mathrm{He}$ shell of the $\mathrm{CO} \mathrm{WD}$ in the following reason. The shock wave emerges in a region with density of $\sim 10^{7} \mathrm{~g} \mathrm{~cm}^{-3}$. In front of the shock wave, there is a region with density of $\sim 5 \times 10^{6} \mathrm{~g} \mathrm{~cm}^{-3}$ and with size of $5 \times 10^{5} \mathrm{~cm}$. This is consistent with the conditions of He detonation obtained by Holcomb et al. (2013). Note that our 1D mesh simulation has sufficiently high resolution $\left(\sim 10^{4} \mathrm{~cm}\right)$ to resolve the required size $\left(\sim 10^{5} \mathrm{~cm}\right)$. As described above, the $1 \mathrm{D}$ mesh simulation overestimates density by about $20 \%$ at the edge of the WD where the shock wave and He detonation wave appear. It underestimates the size of a hotspot required to generate a detonation wave by a factor of 2 , since the size of a hotspot to generate a detonation wave increases by three orders of magnitude with density decreasing by an order of magnitude (Holcomb et al. 2013). The size of a region heated by the shock wave is $5 \times 10^{5} \mathrm{~cm}$, while the size of a hotspot required to generate a detonation wave would be $<5 \times 10^{5} \mathrm{~cm}$. Eventually, the density overestimate in the 1D mesh simulation does not affect the initiation of the $\mathrm{He}$ detonation wave.

On the other hand, the shock wave fails $\mathrm{CO}$ detonation in the CO WD without a He shell. The shock wave emerges in the same position as that in the CO WD with a He shell. However, the composition at the position is $\mathrm{CO}$ composition, not He composition. According to Seitenzahl et al. (2009), $\mathrm{CO}$ detonation succeeds under density environment of $\sim 5 \times$ $10^{6} \mathrm{~g} \mathrm{~cm}^{-3}$ only if a hotspot size is $\gtrsim 10^{6} \mathrm{~cm}$. However, the shock wave heats a region whose size is several $10^{5} \mathrm{~cm}$. Our results are consistent with Seitenzahl et al. (2009).

In the $\mathrm{CO}$ WD with a He shell, the He detonation directly drives the CO detonation. This is so-called "edge-lit" type of the double detonation scenario in the context of type Ia supernovae. For the edge-lit type, the altitude of the initiation point of He detonation from the $\mathrm{CO}$ core-He shell interface should be $\gtrsim 10^{7} \mathrm{~cm}$ (Moll \& Woosley 2013). On the other hand, the edge-lit CO detonation succeeds in our model despite that the altitude is several $10^{5} \mathrm{~cm}$ (see the bottom panel at $t_{1 \mathrm{D}}=0.0376 \mathrm{~s}$ ). We can resolve this discrepancy considering the motion of the CO core-He shell interface. The interface in the case of Moll \& Woosley (2013) is at rest, while the interface in our model proceeds toward the He detonation at a speed of $>5 \times 10^{8} \mathrm{~cm} \mathrm{~s}^{-1}$ (see the middle and bottom panels at $t_{1 \mathrm{D}}=0.0376 \mathrm{~s}$ and $0.0381 \mathrm{~s}$ ). Therefore, the edge-lit CO detonation in the CO core is easier to occur in our model than in the case of Moll \& Woosley (2013). 

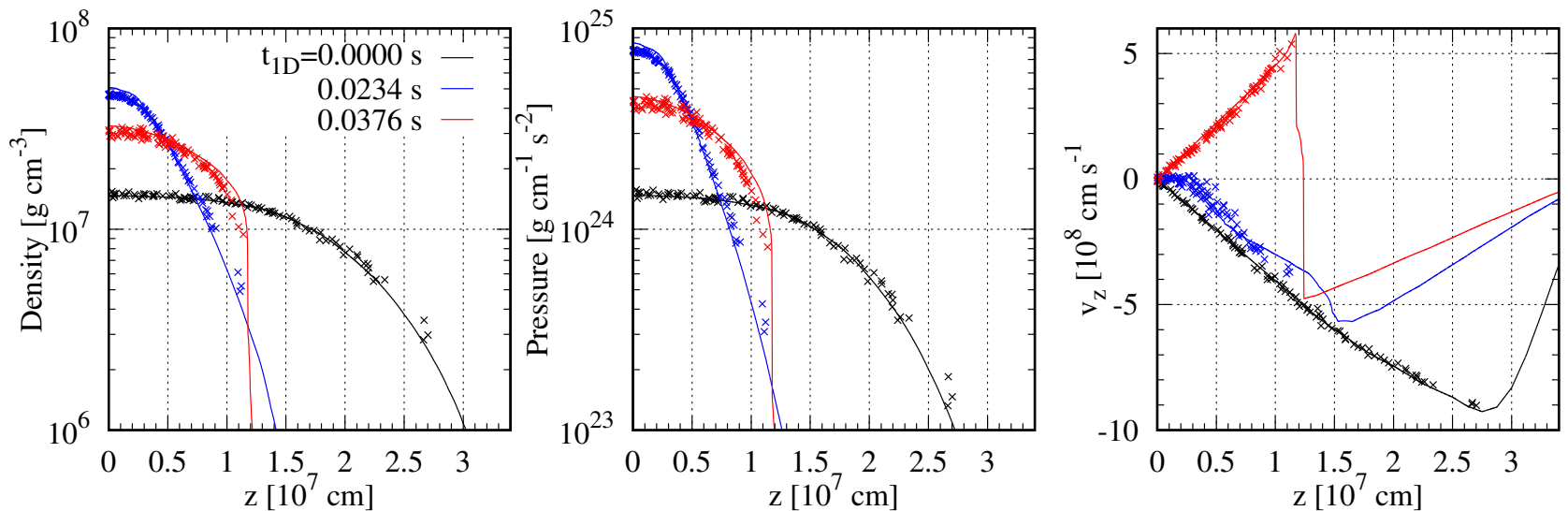

Figure 2. Time evolution of density, pressure, and z-velocity in 1D mesh simulation for a CO WD without a He shell (solid curves) and in 3D SPH simulation (cross points). The cross points indicate physical quantities of SPH particles sampled randomly from the portion. At $t_{1 \mathrm{D}} \sim 0.0234 \mathrm{~s}$, the portion bounces back, and at $t_{1 \mathrm{D}} \sim 0.0376 \mathrm{~s}$, a shock wave appears.

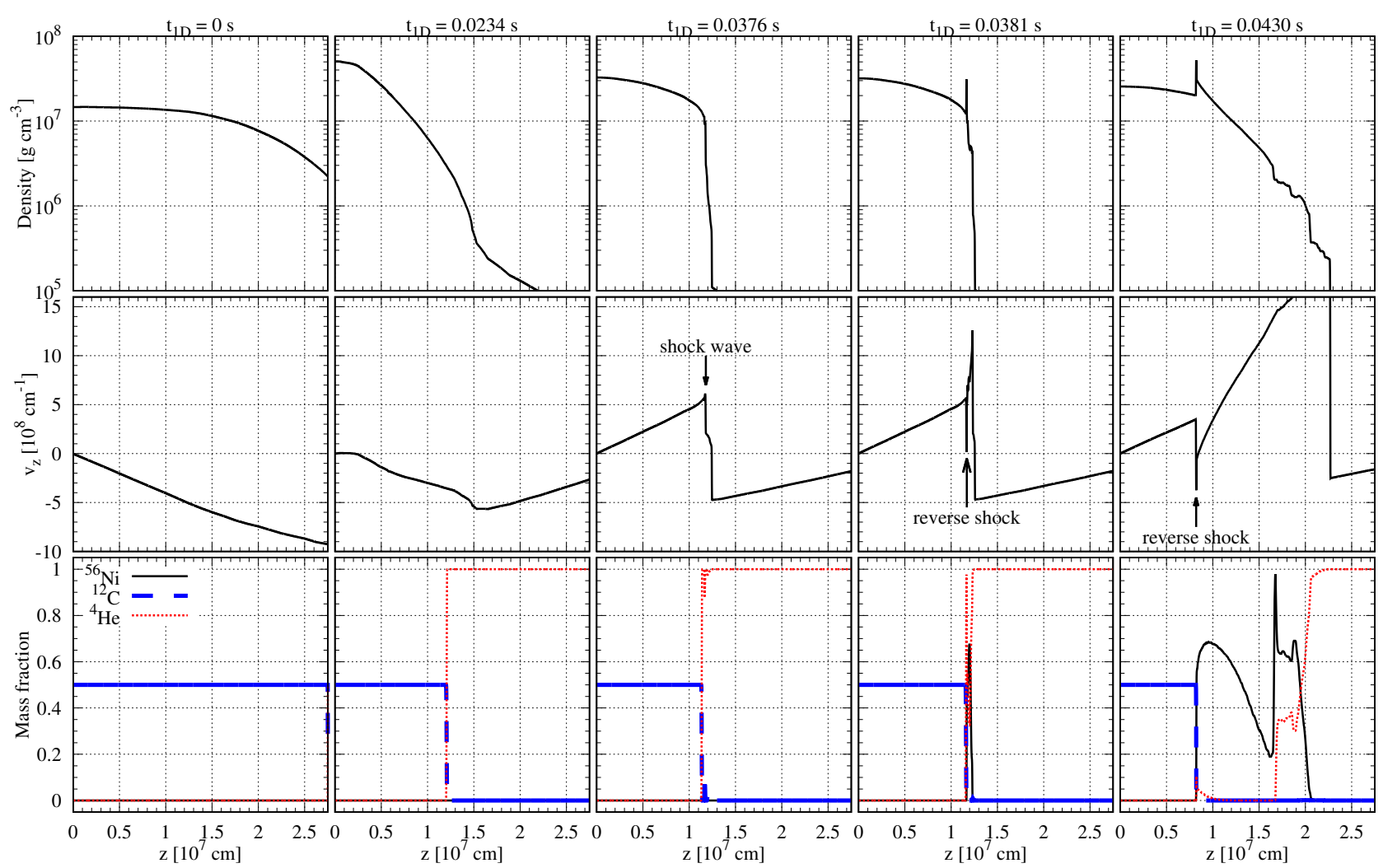

Figure 3. Time evolution of density, $z$-velocity, and nuclear element profiles in $1 \mathrm{D}$ mesh simulation for a CO WD with a He shell. The mass fraction of the He shell is $2 \%$. The initial condition is based on 3D SPH simulation of a CO WD with $N=100 \mathrm{M}$.

\section{CONCLUSION}

We show tidal double detonation actually occurs in a WD TDE by numerical simulation. The WD is a CO WD with a He shell whose mass fraction is at most 5\%. Importantly, if CO WDs with and without a He shell are in the same orbit around an IMBH, tidal double detonation in the CO WD with a He shell is easier to arise than tidal detonation in the CO WD without a He shell. In other words, tidal double detonation emerges for smaller $\beta$ than tidal detona- tion. This means tidal double detonation spreads opportunity to TDEs illuminating IMBHs.

We may distinguish tidal double detonation from simple tidal detonation by the presence of surface radioactivity from ${ }^{56} \mathrm{Ni}$ synthesized by He detonation. As seen in the bottom panel at $t_{1 \mathrm{D}}=0.0430$ in Figure 3 , He detonation yields larger mass fraction of ${ }^{56} \mathrm{Ni}$ than $\mathrm{CO}$ detonation under similar density environments $\left(\sim 10^{7} \mathrm{~g} \mathrm{~cm}^{-3}\right)$. Therefore, WD TDEs powered by tidal double detonation have earlier emission than those powered by simple tidal detonation, analogously to type Ia supernovae possibly with He detona- 


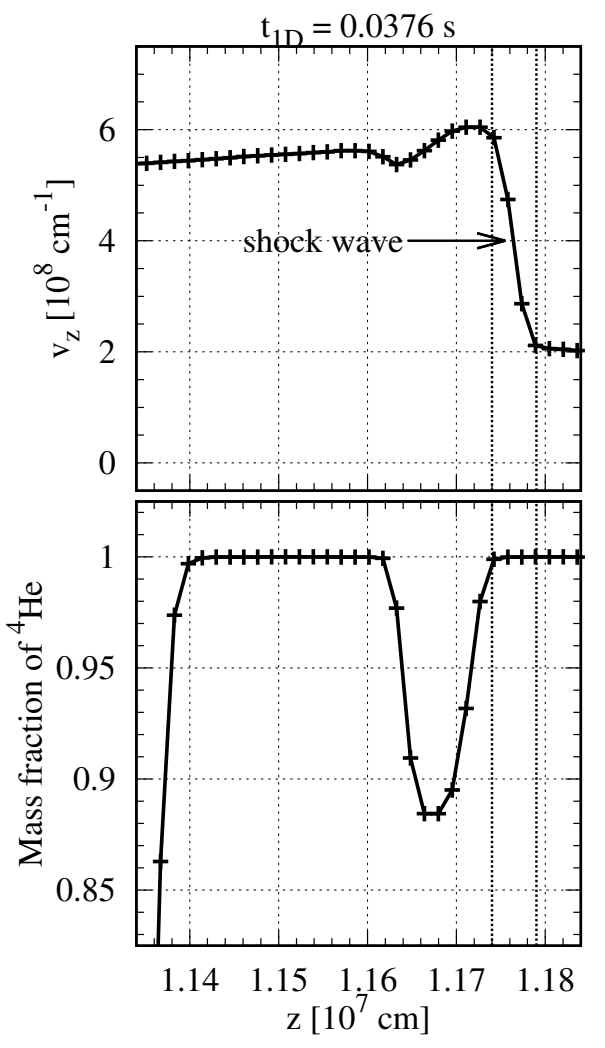

Figure 4. Profiles of $z$-velocity and nuclear components at $t_{1 \mathrm{D}}=$ $0.0376 \mathrm{~s}$ where the He detonation starts. Cross points indicate positions of cells in 1D mesh simulation. The shock wave indicated in Figure 3 is between two vertical dotted lines.

tion (e.g. Diehl et al. 2014; Kromer et al. 2016; Jiang et al. 2017).

\section{ACKNOWLEDGMENTS}

A. Tanikawa thanks I. Hachisu for fruitful discussions. Numerical computations were carried out on Cray XC30 at Center for Computational Astrophysics, National Astronomical Observatory of Japan, on Cray XC40 at Yukawa Institute for Theoretical Physics, Kyoto University, and on Oakforest-PACS at Joint Center for Advanced High Performance Computing. The software used in this work was in part developed by the DOE NNSA-ASC OASCR Flash Center at the University of Chicago. This research has been supported in part by MEXT program for the Development and Improvement for the Next Generation Ultra High-Speed Computer System under its Subsidies for Operating the Specific Advanced Large Research Facilities, and by Grants-inAid for Scientific Research (16K17656, 17H06360) from the Japan Society for the Promotion of Science.

\section{REFERENCES}

Auchettl K., Guillochon J., Ramirez-Ruiz E., 2017, ApJ, 838, 149 Bildsten L., Shen K. J., Weinberg N. N., Nelemans G., 2007, ApJ, 662, L95

Diehl R., et al., 2014, Science, 345, 1162
Fryxell B., et al., 2000, ApJS, 131, 273

Guillochon J., Dan M., Ramirez-Ruiz E., Rosswog S., 2010, ApJ, 709, L64

Holcomb C., Guillochon J., De Colle F., Ramirez-Ruiz E., 2013, ApJ, 771, 14

Iben Jr. I., Tutukov A. V., 1985, ApJS, 58, 661

Iben Jr. I., Tutukov A. V., 1993, ApJ, 418, 343

Iwasawa M., Tanikawa A., Hosono N., Nitadori K., Muranushi T., Makino J., 2016, PASJ, 68, 54

Jiang J.-A., et al., 2017, Nature, 550, 80

Komossa S., 2015, Journal of High Energy Astrophysics, 7, 148

Kromer M., et al., 2016, MNRAS, 459, 4428

Livne E., 1990, ApJ, 354, L53

Luminet J.-P., Pichon B., 1989, A\&A, 209, 103

Moll R., Woosley S. E., 2013, ApJ, 774, 137

Nomoto K., 1980, in Wheeler J. C., ed., Texas Workshop on Type I Supernovae. pp 164-181

Nomoto K., 1982, ApJ, 257, 780

Pakmor R., Kromer M., Taubenberger S., Springel V., 2013, ApJ, $770, \mathrm{~L} 8$

Parsons S. G., et al., 2017, MNRAS, 470, 4473

Rosswog S., Ramirez-Ruiz E., Hix W. R., Dan M., 2008, Computer Physics Communications, 179, 184

Rosswog S., Ramirez-Ruiz E., Hix W. R., 2009, ApJ, 695, 404

Salaris M., García-Berro E., Hernanz M., Isern J., Saumon D., 2000, ApJ, 544, 1036

Seitenzahl I. R., Meakin C. A., Townsley D. M., Lamb D. Q., Truran J. W., 2009, ApJ, 696, 515

Shen K. J., Kasen D., Weinberg N. N., Bildsten L., Scannapieco E., 2010, ApJ, 715, 767

Tanikawa A., 2017, preprint, (arXiv:1711.05451)

Tanikawa A., Nakasato N., Sato Y., Nomoto K., Maeda K., Hachisu I., 2015, ApJ, 807, 40

Tanikawa A., Sato Y., Nomoto K., Maeda K., Nakasato N., Hachisu I., 2017, ApJ, 839, 81

Tejeda E., Rosswog S., 2013, MNRAS, 433, 1930

Timmes F. X., Swesty F. D., 2000, ApJS, 126, 501

Timmes F. X., Hoffman R. D., Woosley S. E., 2000, ApJS, 129,377

Wilson J. R., Mathews G. J., 2004, ApJ, 610, 368

Woosley S. E., Weaver T. A., Taam R. E., 1980, in Wheeler J. C., ed., Texas Workshop on Type I Supernovae. pp 96-112

This paper has been typeset from a TEX/LATEX file prepared by the author. 


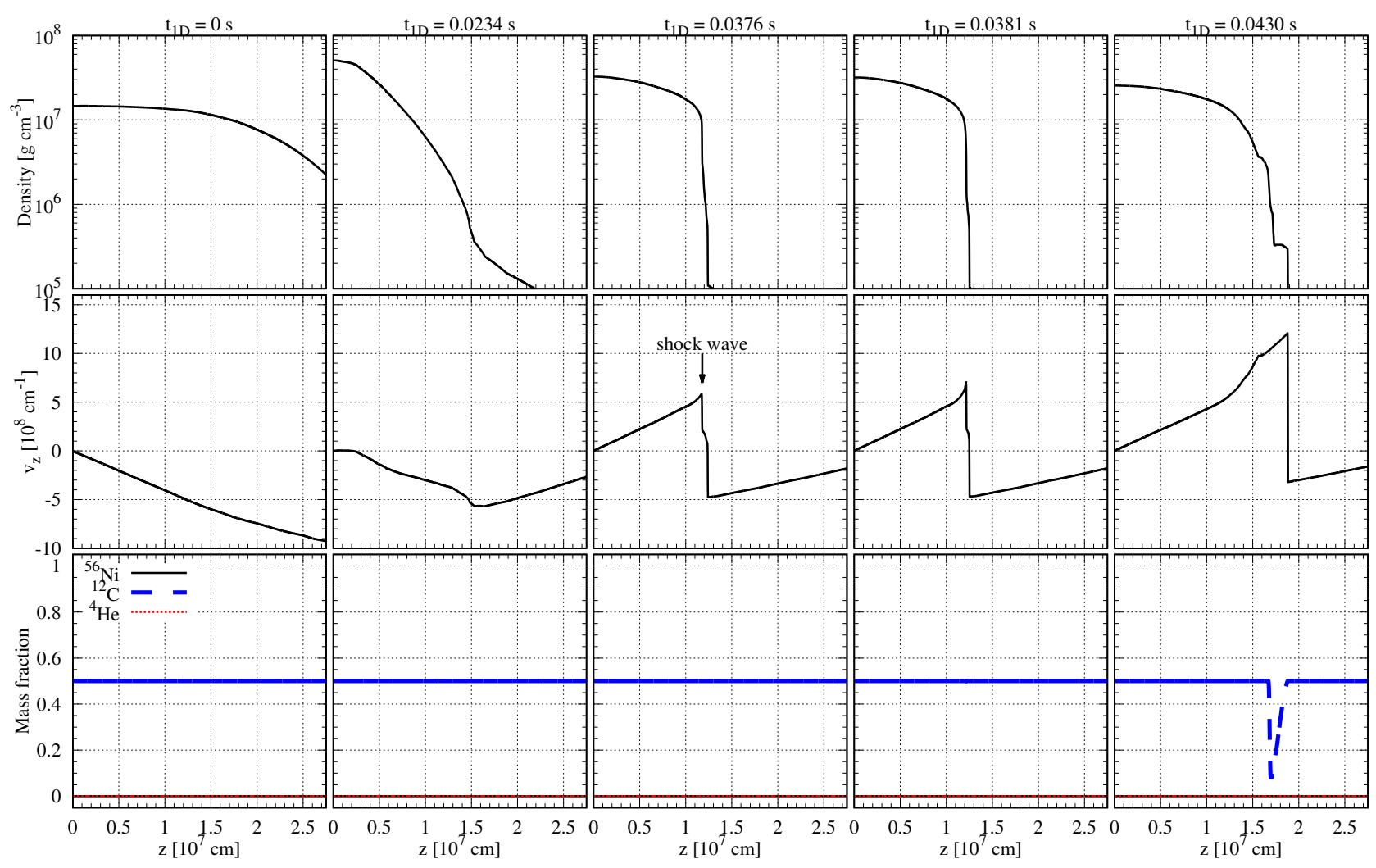

Figure 5. The same as Figure 3, except for a CO WD without a He shell. 